\title{
OPTICAL BEAM TIMING MONITOR EXPERIMENTS AT THE ADVANCED LIGHT SOURCE*
}

\author{
J. Byrd, S. De Santis", R. Wilcox, LBNL, Berkeley, California \\ Y. Yin, YYLabs, Fremont, California.
}

\begin{abstract}
We present the initial results of an experimental study of a beam timing monitor based on an optoelectronic technique. This technique uses the electrical signal from a beam position monitor to modulate the amplitude of a train of laser pulses, converting timing jitter into an amplitude jitter. This modulation is then measured with a photodetector and sampled by a fast ADC. This approach has already demonstrated sub-100 fs resolution and promises even better results. Additionally, we are planning to use the technique as a way to extract the maximum possible bandwidth from a BPM, avoiding the dispersion typical of long RF cables. We show our initial results using signals from the Advanced Light Source storage ring.
\end{abstract}

\section{INTRODUCTION}

The principles of a beam timing monitor using optoelectronic technologies have been presented by $\mathrm{F}$. Loehl et al. in [1].

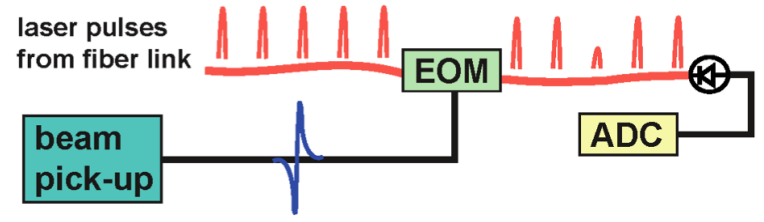

Fig.1. Scheme of principle of an optoelectronic beam timing monitor (courtesy of F. Loehl, et al.).

Figure 1 shows the fundamental components of such a system: the signal from a beam pick-up is used to modulate the amplitude of a train of laser pulses by an electro-optic modulator (EOM). The laser pulses are then detected with a photodiode and their amplitude information is converted into digital format and processed/stored. In order to achieve maximum sensitivity, the laser pulses have to be timed in such a way to be synchronous with the zero crossing of the BPM signal. The steepness of the BPM signal slope (and therefore its bandwidth), together with the laser pulse width and the system signal-to-noise ratio define the ultimate time resolution achievable with this technique. Instead of using a pulsed laser, one can also replace it with a CW laser, still using a similar arrangement for signal detection. With this setup one obtains a bunch profile monitor. In this case the resolution is dominated by the EOM's and the photodiode bandwidth. With the currently available components, which feature 10's of
$\mathrm{GHz}$ bandwidths, one can in principle obtain resolutions of the order of a few 10's of picoseconds, which are more than adequate for hadron storage rings, for instance.

In the next section we describe our experimental setup on the storage ring of the Advanced Light Source.

In the following section we then discuss our results and finally our plans for the continuation of our activity.

\section{EXPERIMENTAL SETUP AT THE ADVANCED LIGHT SOURCE}

The ALS storage ring parameters relevant to our experiment are reported in Tab.1.

Tab.1. Relevant parameters of the ALS storage ring.

\begin{tabular}{|l|l|}
\hline Beam energy $\mathbf{E}_{\mathbf{b}}$ & $1.9 \mathrm{GeV}$ \\
\hline Ring circumference $\mathbf{L}_{\mathbf{c}}$ & $196.8 \mathrm{~m}$ \\
\hline Harmonic number $\mathbf{h}$ & 328 \\
\hline Filling pattern & $276 / 328+1$ \\
\hline Bunch length $\boldsymbol{\sigma}_{\text {FWHM }}$ & $70 \mathrm{ps}$ \\
\hline Total beam current $\mathbf{I}_{\mathbf{b}}$ & $400-200 \mathrm{~mA}$ \\
\hline
\end{tabular}

Two complete sets of button BPM's were available and we managed to pass a few 1/4-inch Heliax cables and standard SMA cables through a gap in the ceiling shielding. In this way we minimized the cables length, to less than 2 meters. The beam signal available at the end of the cable is in the order of around $2 \mathrm{~V}$ at full beam current.

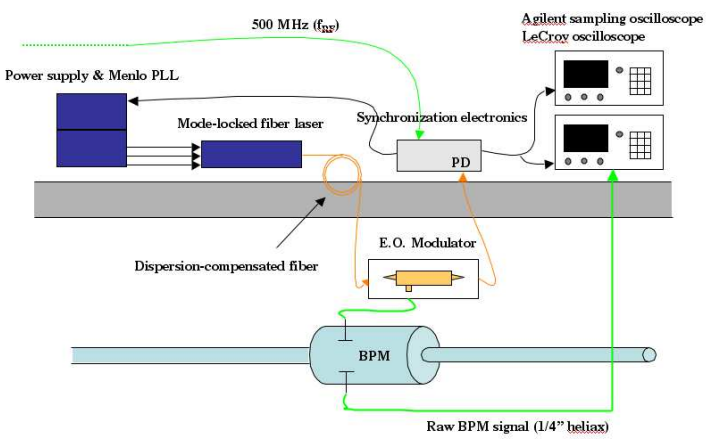

Fig.2. Experimental setup schematics.

*Work supported by the U.S. Department of Energy under Contract No. DE-AC0-05CH11231.

"sdesantis@1bl.gov 


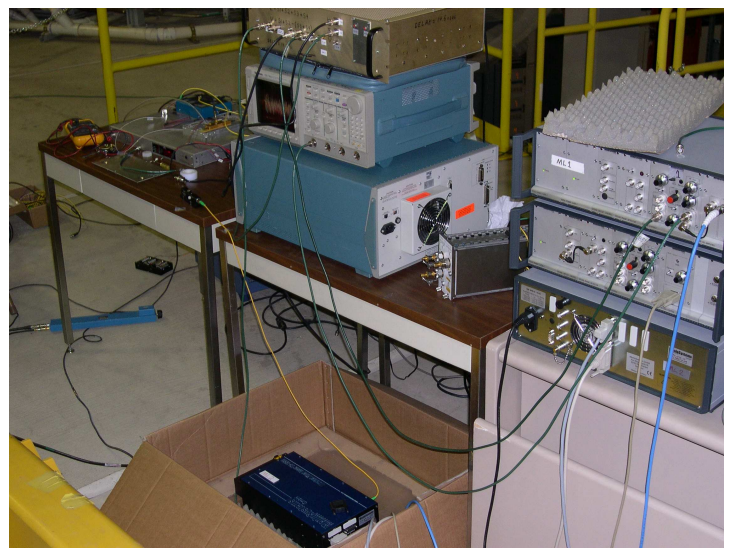

Fig.3. Experimental setup at the ALS.

As a first stage of our experiment, we placed the entire instrumentation on top of the storage ring tunnel roof, so that all the electronics was always available for troubleshooting, without the necessity of any controlled access. Figure 2 and 3 show the instrumental setup in its final configuration, once we place the electro-optic modulator inside the tunnel, as close as possible to the beam position monitor SMA connector.

We will retain some of the RF cables for diagnostic purposes. We used a JDSU $10 \mathrm{~Gb} / \mathrm{s}$ modulator. The laser we intend to use for beam timing is a MenloSystems $\mathrm{GmbH}$ TC1550 $1550 \mathrm{~nm}$ wavelength fiberlaser. It has a $100 \mathrm{MHz}$ repetition frequency, which would allow to sample every fifth bunch of the ALS after being phase locked to the ring's RF frequency.

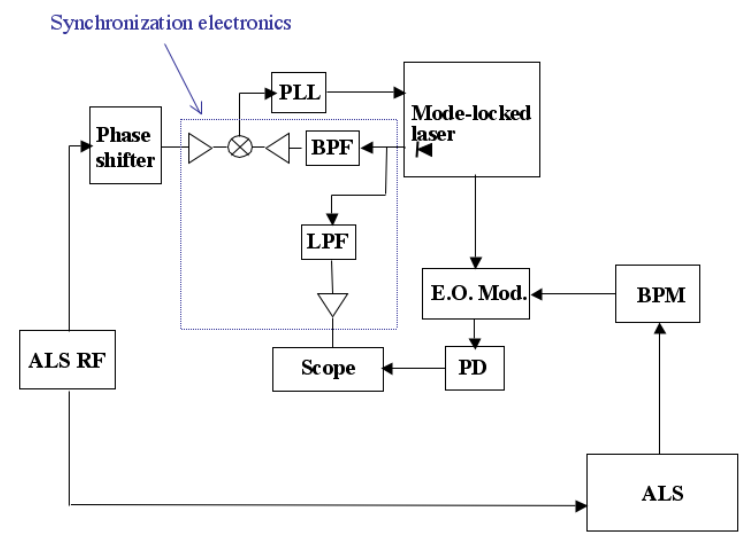

Fig.4. Block diagram of the experimental setup.

A block diagram of all the components is shown in Fig.4. The fiber laser has an internal photodiode, the output of which is used as both a $100 \mathrm{MHz}$ local oscillator for synchronization to the main RF and as a trigger for our oscilloscope synchronous with the laser pulses.

For laser pulse detection we experimented with several devices, as well as with an Agilent $53 \mathrm{GHz}$ sampling scope with an 86116A optical input module. We obtained our best results by using an InGaAs $5 \mathrm{GHz}$ bandwidth PIN diode (Discovery Semiconductors Inc. DSC50).

Since the fiber laser was available to us only for a short time, we carried on most of our initial measurements using a $1550 \mathrm{~nm} \mathrm{CW}$ laser, in order to test our components and find a suitable photodiode.

We are planning to install the modulator inside the tunnel and return to use the pulsed laser. We are also planning to acquire a $9 \mathrm{GHz}$ PIN diode (DSC30) to be able to detect the maximum possible bandwidth out of our BPM's.

\section{INITIAL EXPERIMENTAL RESULTS}

Although we were able to detect a beam signal with a relatively small effort, our time for data taking was cut short by the upcoming seasonal long shutdown for the ALS.

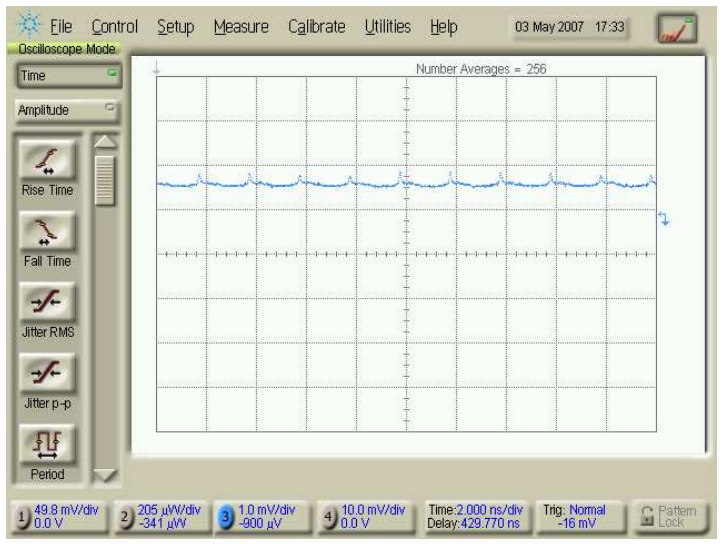

Fig.5. 2 ns spaced ALS bunches. The BPM signal modulates a CW laser.

As stated earlier, we eventually took our measurements using a CW laser. Figure 5 shows the output of the Agilent $53 \mathrm{GHz}$ sampling scope with an integrate optical module. The ALS bunches, spaced at $2 \mathrm{~ns}$ intervals are clearly visible.

In order to increase our signal-to-noise ratio we experimented with a variety of photodetectors we had available, obtaining the best result with the DSC50 InGaAs PIN diode described in the previous section.

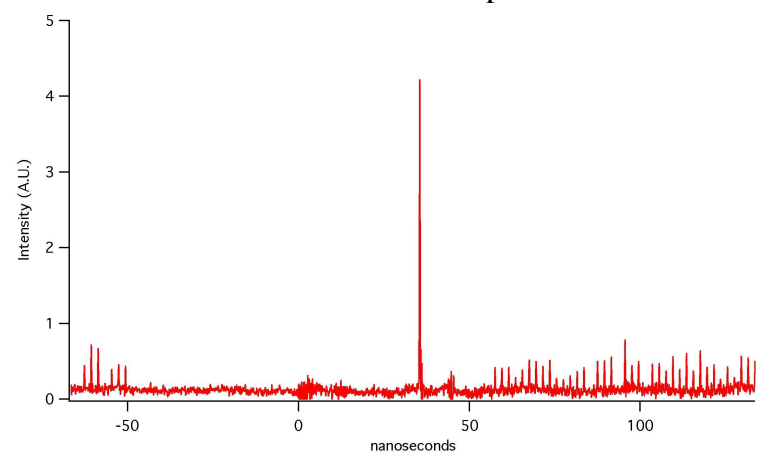

Fig.6. ALS fill gap with camshaft bunch

Figure 6 shows the beam signal detected using the DSC50 diode. Signal-to-noise ratio is much improved. The ALS features a higher current bunch (camshaft) inside a $104 \mathrm{~ns}$ gap in the fill.

We could estimate the time resolution of our system by comparing the bunch length measured for the camshaft, where we get the strongest signal with its known value. 


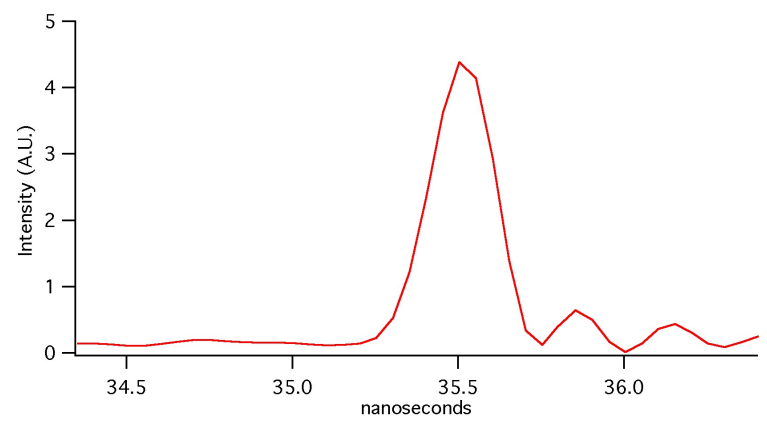

Fig.7. Detail of the ALS camshaft bunch.

From Fig.7 we can see how the FWHM bunch length, as measured by our system is equal to approximately 200 ps. From the ALSA diagnostic beamline (using a streak camera) we have independently measured the FWHM around $70 \mathrm{ps}$. Since the bandwidth of the photodiode used is $5 \mathrm{GHz}$, this would justify the discrepancy between the two measurements.

\section{CONCLUSIONS}

We have shown our first measurements at the ALS of a bunch monitor that makes use of an optoelectronic technique. Although we were not able to take data with the pulsed laser, as we intended, by using a CW laser we could test most of our electronics, synchronize our instrumentation to the ALS $499.645 \mathrm{MHz}$ main RF frequency and identify a family of InGaAs PIN photodiodes suitable for our applications. The time resolution of the measurements taken to-date is limited by the $5 \mathrm{GHz}$ photodiode we are currently using.

In the near future, we plan to restore the original configuration with the ManloSystems $100 \mathrm{MHz}$ pulsed fiberlaser and solve the problems connected with sampling a beam the bunch frequency of which is not an integer multiple of $100 \mathrm{MHz}$. Additionally, now that we have confidently tested it, we will move our electro-optic modulator inside the storage ring tunnel, as close as possible to the BPM connector. By doing this and after acquiring a faster photodiode, we will be able to increase the bandwidth of the bunch profile measurements.

\section{REFERENCES}

[1] F. Loehl, K. Hacker, et al., A Sub 100 fs Electron Bunch Arrival-Time Monitor System for FLASH, in Proc. of EPAC 2006, Edinburgh, 2006. 